\title{
Enhancement of Green Fluorescent Protein Expression in Adeno-Associated Virus with the Woodchuck Hepatitis Virus Post-Transcriptional Regulatory Element
}

\author{
Jonathan E. Loeb, Matthew D. Weitzman, and Thomas J. Hope
}

\section{Introduction}

Since the advent of recombinant DNA technology, maximization of exogenous gene expression has been an important issue for molecular biologists. Efforts at enhancing transgene expression have mostly been directed at improving the efficiency of delivery and increasing levels of transcription and translation. Less progress has been made in the application of post-transcriptional methods for improving gene expression. Here is described the use of an element derived from the woodchuck hepatitis virus (WHV) that possesses the ability to enhance the expression of heterologous genes post-transcriptionally. Green fluorescent protein (GFP) is frequently employed as a fusion protein to enable the detection, visualization, and quantification of molecules under study. Problems of expression are often encountered when using this strategy, making the woodchuck hepatitis virus post-transcriptional regulatory element (WPRE) a useful addition to vectors designed to express fusion proteins. This chapter discusses general issues of cloning and placement of the WPRE when designing such vectors.

Gene expression can be modulated at many levels, including transcription, post-transcriptional modification of RNA such as $5^{\prime}$ and $3^{\prime}$ end-processing, RNA export and stability, and translation. Additionally, the presence of introns, or the process of intron splicing itself, can increase gene expression (1-3). New insight continues to be acquired regarding how viruses ensure high levels of expression of their genes at the post-transcriptional level. Several viruses, 
including HIV, type D retroviruses, murine leukemia virus, cytomegalovirus (CMV), influenza virus, the herpesviruses, and the hepatitis B viruses, have been shown to possess cis- and trans-acting elements whose purpose is to posttranscriptionally increase the expression of viral genes by interacting with hostcell machinery (4-10). In some cases, such elements are supporting the expression of intronless viral genes. The expression of heterologous cDNAs, which are intronless messages, for experimental purposes, could likewise be enhanced by such elements. The post-transcriptional regulatory element of hepatitis B virus (HPRE) can functionally replace an intron in stimulating $\beta$-globin expression (11). Our studies of the WHV demonstrated that it contains a post-transcriptional regulatory element (WPRE), which is partially homologous to the HPRE (5). These PREs function in an orientation-dependent manner, suggesting that they are RNA elements. Consistent with this interpretation, the PREs contain conserved RNA stem-loop structures, which are required for maximal function, and whose structure has been confirmed by compensatory mutational analysis (12). The WPRE functions more efficiently than the HPRE, because of the presence of an additional cis-acting sequence in the WPRE, termed " $\gamma$ " (5). The mechanism of this enhancement, although incompletely understood, is known to be post-transcriptional in nature and may involve the facilitation of RNA processing and/or export $(13,14)$.

Our studies reveal that the post-transcriptional effect of the WPRE can significantly stimulate the expression of GFP in transient and stable transfections (Fig. 1; 15). Increases in GFP expression of greater than twofold are obtainable in 293 cells transiently transfected with GFP-expressing plasmids, 6-9-fold increases in expression have been obtained in the context of stably transfected 293 cells or primary human fibroblasts, as well as in other primary tissue (15). We surmise that this differential effect may result from the comparatively large number of plasmid copies being expressed in the context of transient, as opposed to stable, transfection, in which cells can contain as few as one integrated copy of the heterologous gene. In the transient context, high copy number may saturate those pathways of RNA metabolism influenced by the WPRE, thus minimizing its effect. The WPRE has also been shown to enhance expression in RNA and DNA viral vectors, including murine leukemia virus, HIV (Fig. 2), and adenoassociated virus (AAV), using various promoters and transgenes $(\mathbf{1 5}, 17)$. The WPRE effect also appears to be species-independent, as evidenced by work in murine and avian cells. Thus, the WPRE is a broadly useful tool for increasing gene expression. Here, is described in detail the use of the WPRE in enhancing GFP expression in the context of AAV. Protocols are provided for preparing plasmids that express GFP, with and without the WPRE in the message, and for adapting them for use in an AAV shuttle vector. We further discuss production of recombinant AAV stocks, and verification of enhanced expression. 


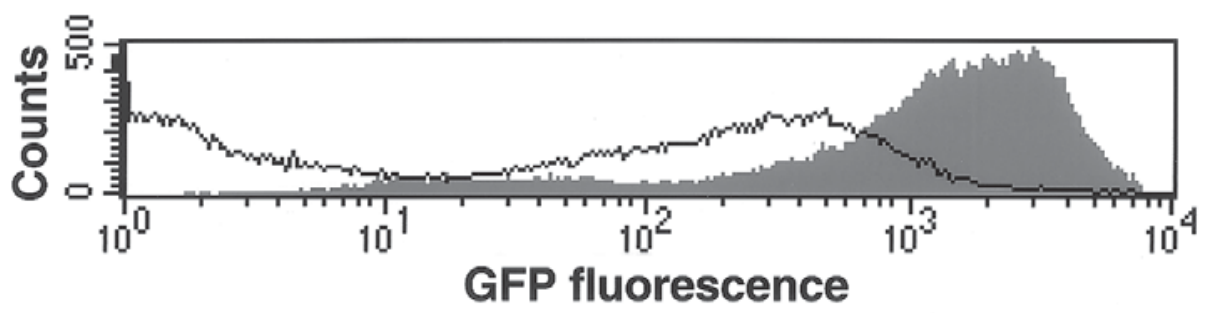

\section{+WPRE Geometric Mean 863.05 -WPRE Geometric Mean 34.99}

Fig. 1. WPRE enhances GFP expression in 293 cells stably transfected with GFPexpressing constructs. 293 cells were transfected with plasmids that included (+WPRE), or did not include (-WPRE), the WPRE in messages expressing GFP driven by the CMV promoter. Following selection in media containing $200 \mu \mathrm{g} / \mathrm{mL}$ G418 for 4 wk, 30,000 cells were analyzed for GFP expression by flow cytometry. Geometric mean fluorescence is shown for each population.

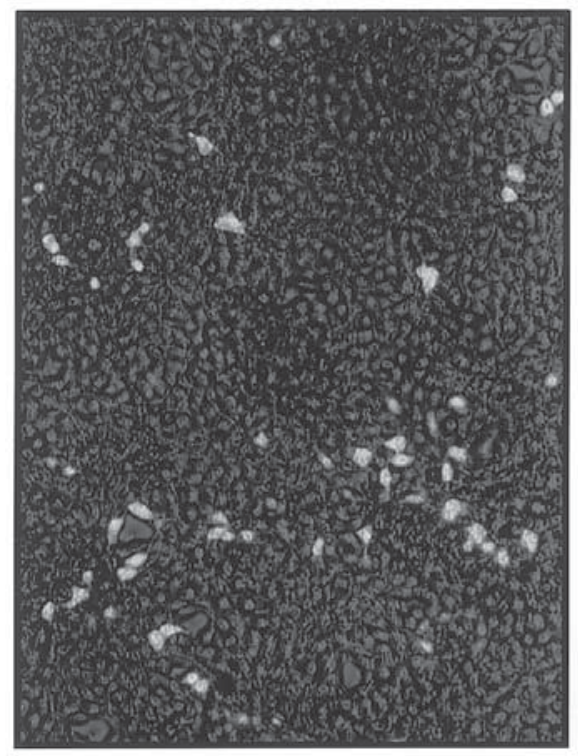

pHR'cmvGFP WHV

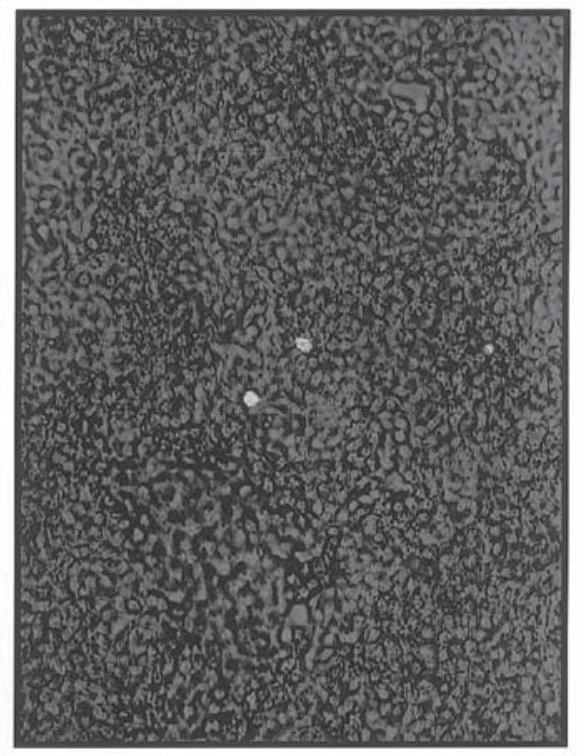

pHR'cmvGFP

Fig. 2. WPRE enhances GFP expression in 293 cells transduced with lentiviral vectors. 293 cells were transduced with HIV-1-based vectors which included (+WPRE), or did not include (-WPRE), the WPRE in messages expressing GFP driven by the HIV-1 long terminal repeat (LTR). Cells were visualized by fluorescence microscopy. Only high-expressing cells are visualized here. 


\section{Materials}

1. WPRE sequence: The WPRE has been functionally defined as encompassing nucleotides of position 1093-1684 of WHV 8 (5). The viral DNA template of WHV 8 is available from the American Type Culture Collection (Genbank accession number J04514).

2. Plasmids: This sequence is available as a subclone in the plasmid pSK+WPREB11 (5). The plasmid contains the WPRE inserted at the ClaI site (see Note 1). Also needed for this protocol are EGFP-C1 (Clontech), psub201 (18), rep/cap packaging plasmid and adenovirus helper plasmid for AAV production (16).

3. Primers: If direct subcloning of the WPRE into the expression vector by restriction digest is not possible, the WPRE can be amplified from pSK+WPRE-B11 or from the WHV8 genome, using the following primers (see Note 2). 5'-AATCAACCTCTGGATTACAAAAT-3' and 5'-AGGCGGGGAGGCGGCCCAAAGGGA-3'. Other synthetic oligonucleotides as listed in Subheading 3.

4. Enzymes: Restriction enzymes desribed here are obtained from New England Biolabs, and the buffers used are those recommended by the manufacturer. Required are: BglII, EcoRI, SspI, XbaI, BspHI, AseI, ClaI; buffers 2, 3, 4, EcoRI, $S s p \mathrm{I}$; bovine serum albumin, provided with enzymes. Also needed are calf intestinal phosphatase (CIP) and its buffer (Gibco/Life Technologies) and T4 DNA ligase and its buffer (Gibco/Life Technologies).

5. Cell culture and viral preparation: Dulbecco's modified Eagle medium (DMEM) (BioWhittaker), fetal bovine serum (Gibco/Life Technologies) and penicillinstreptamycin-L-glutamine solution (BioWhittaker). For transfection: $2.5 \mathrm{M} \mathrm{CaCl}_{2}$, 2X HEPES-buffered saline (20 mM HEPES, pH 7.8, $150 \mathrm{~m} M \mathrm{NaCl}$ ), $10 \mathrm{~m} M$ Tris-Cl, pH 7.6, DNaseI (Ambion) and RNase A $10 \mathrm{mg} / \mathrm{mL}$ (Ambion), $4 M$ ammonium sulfate, $\mathrm{CsCl}, 10 \%$ glycerol, HybQuest kit (Mirus) for dot blotting, $200 \mu \mathrm{g} / \mathrm{mL}$ Geneticin G418 (Gibco/Life Technologies), phosphate-buffered saline (PBS) (BioWhittaker), and PBS $5 \mathrm{~m} M$ EDTA, $5 \mathrm{~m} M$ propidium iodide.

\section{Methods}

A generally applicable strategy for using the WPRE in an expression vector consists of the digestion of pSK+WPRE-B 11 at the appropriate restriction sites to allow the insertion of the WPRE into the expression plasmid. Alternatively, subcloning can be achieved by polymerase chain reaction amplification of the WPRE, using primers with convenient restriction sites incorporated at their 5 ' ends, followed by digestion and insertion into the target plasmid at the appropriate site (see Note 3 ).

\subsection{Production of AAV Plasmids Containing WPRE for EGFP Expression}

Plasmids used in these transfection and transduction experiments are based on the EGFP-C1 vector (Clontech). Since the vector was originally intended 
for the construction of eGFP fusion proteins, a stop codon must be inserted after the GFP reading frame, to allow the use of the multiple cloning site for the addition of untranslated elements. For maximum flexibility, the stop codon may be triple-frame.

1. Digest EGFP-C1 (Clontech) with BglII and EcoRI in EcoRI buffer (New England Biolabs). Isolate the opened plasmid by gel electrophoresis.

2. Insert the triple-frame stop as a pair of annealed synthetic oligonucleotides of sequences GATCTTAGCTAACTG (sense) and AATTCAGTTAGCTAA (antisense). The oligos may be annealed by incubating an equimolar mixture of the pair in $0.5 \mathrm{M} \mathrm{NaCl}$ for $2 \mathrm{~min}$ at $95^{\circ} \mathrm{C}$ followed by $5 \mathrm{~min}$ at $65^{\circ} \mathrm{C}, 10 \mathrm{~min}$ at room temperature, and $5 \mathrm{~min}$ on ice. The annealed fragment will possess sticky ends compatible with with $B g l \mathrm{II}$ and $E c o$ RI, and can be ligated directly into the EGFP-C1 vector, using T4 DNA ligase (Gibco/Life Technologies) according to the manufacturer's recommendations to produce the plasmid EGFP.stop. Transform Escherichia coli, and select on plates containing kanamycin; this and all subsequent selection of bacteria should be performed with kanamycin.

3. Optimum packaging of AAV occurs with an insert size of approx $4.5 \mathrm{~kb}$. To adapt the construct for use in AAV, cut the plasmid, EGFP.stop, with $S s p I$ in $S s p$ I buffer, to remove the $\mathrm{f} 1$ ori and ampicillin resistance promoter, and religate with T4 DNA ligase, reducing the size of the recombinant AAV genome eventually generated, and producing plasmid eGFP.stop. $\Delta$ Ssp.

4. To allow transfer of the expression cassette to the AAV shuttle vector, psub201 (18), insert synthetic linkers consisting of oligonucleotides annealed as before, containing $X b a I$ restriction sites and sticky ends compatible with the unique $B s p H I$ (oligo sequences: CATGACGTCTAGACG and CATGCGTCTAGACGT) and AseI (oligo sequences: TAATCGTCTAGACG and TACGTCTAGACGAT) sites in EGFP.stop. $\triangle$ Ssp, yielding EGFP.stopBXAX. These insertions must be performed one at a time in series; digest eGFP.stop. $\Delta$ Ssp with $B s p \mathrm{HI}$ in buffer 4, with AseI in buffer 3. The oligos are designed so that one of the $B s p \mathrm{HI}$ or AseI sites flanking the $\mathrm{XbaI}$ will be destroyed. Therefore, at this stage, sequencing through the subcloning junctions is useful, to ascertain the orientation of the artificial $\mathrm{Xba \textrm {I }}$ site inserts. Additionally, if a control construct is desired in which the WPRE is introduced upstream of the CMV promoter so that it is included in the AAV insert, but not in the CMV-driven message (see step 7), then it is important to use a plasmid in which AseI is intact $3^{\prime}$ to the $X b a \mathrm{I}$ site.

5. Open plasmid EGFP.stopBXAX with $A c c \mathrm{I}$ in buffer 4 , and treat the plasmid with CIP (Gibco/Life Technologies) according to the manufacturer's recommendations. Cut plasmid pSK+WPRE-B11 with ClaI in buffer 4+BSA, isolate the resulting 591 base fragment comprising the WPRE by gel electrophoresis, and ligate into the EGFP.stop.BXAX vector at the $A c c \mathrm{I}$ site, to produce the plasmid EGFP.stop.WPRE. Once again, orientation of the insert must be ascertained by sequencing or digestion. 
6. Open psub201 by digestion with $X b a \mathrm{I}$ in buffer $2+$ bovine serum albumin. Treat with CIP as directed by the manufacturer. Excise the expression cassettes from EGFP.stop.BXAX and eGFP.stop.WPRE by digestion with XbaI and purify by gel electrophoresis. Ligate the cassettes, with and without WPRE, into the XbaI site of the AAV shuttle vector psub201, yielding pAAV.EGFP.W and pAAV.EGFP.stop.

7. To generate a control plasmid, pAAV.EGFP.W(as), in which the WPRE is introduced upstream of the CMV promoter in the antisense orientation, so that it included in the AAV insert but not in the CMV-driven message, bases 10931684 of WHV8 can be amplified by polymerase chain reaction to yield a product with AseI restriction sites at each end, using synthetic oligos of sequences CGCGATTAATAATCAACCTCTGGATTACAAAAT and CGCGATTAATAGGCGGGGAGGCGGCCCAAAGGGA. The PCR product can then be cut with AseI in buffer 3 and ligated into the AseI site of the psub201.EGFP.stop vector. Orientation of the insert should be confirmed by sequencing or digestion. All plasmids in the psub201 vector should be grown in recA' E. coli HB101 to prevent recombination caused by the presence of the inverted terminal repeats.

\subsection{Generation of Purified Recombinant AAV Stocks}

1. Seed $10 \times 10$-cm plates with 293 cells in DMEM supplemented with $10 \%$ fetal bovine serum and $1 \%$ penicillin-streptamycin-L-glutamine solution so that they will be at $90-95 \%$ confluence at the time of transfection. Allow the cells to recover at least $24 \mathrm{~h}$ after passing and prior to transfection.

2. Change the media on the plates for $10 \mathrm{~mL}$ fresh media and place back into the incubator for at least $1 \mathrm{~h}$ to allow for $\mathrm{pH}$ stabilization.

3. Prepare the transfection solution. The conditions for each $10-\mathrm{cm}$ plate should be a total of $25 \mu \mathrm{g}$ plasmid DNA in a 1:1:3 ratio (psub201.eGFP.stop/WPRE: rep/cap packaging plasmid: adenovirus helper plasmid). The transfection cocktail conditions for each plate are then as follows: $5 \mu \mathrm{g}$ psub201.eGFP.stop/WPRE, $5 \mu \mathrm{g}$ rep/cap packaging DNA, $15 \mu \mathrm{g}$ Adenovirus helper plasmid, $60 \mu \mathrm{L} 2.5 \mathrm{M}$ $\mathrm{CaCl}_{2}, 440 \mu \mathrm{L}$ distilled $\mathrm{H}_{2} \mathrm{O}, 500 \mu \mathrm{L} 2 \mathrm{X}$ HEPES, for a total volume of $1 \mathrm{~mL}(\mathbf{1 6})$.

4. Add the plasmid DNA to the $\mathrm{CaCl}_{2}$ and $\mathrm{H}_{2} \mathrm{O}$, and mix well. In a separate polystyrene tube, place the $2 \mathrm{X}$ HEPES. Add the $\mathrm{CaCl}_{2}$ mixture drop-wise to the HEPES, while vortexing. Allow this to sit undisturbed for 5-15 min and monitor the precipitate formation.

5. Remove 1-2 10-cm plates at a time from the incubator to keep the $\mathrm{pH}$ as stable as possible and add $1 \mathrm{~mL}$ of the cocktail to each plate, drop-wise, distributing evenly over all of the plate and agitating gently to mix into the media. Place the plates back into the incubator as soon as possible.

6. About $15-20 \mathrm{~h}$ posttransfection, remove the media, wash once with PBS, then add fresh DMEM. Allow the cells to produce virus for 48-72 h.

7. The cells should easily detach from the plate at the time of harvest by pipeting. Collect in a centrifuge tube and spin the cell suspension in the range of $1500 \mathrm{~g}$ for 
15-20 min at $4^{\circ} \mathrm{C}$. Spinning the cells at too high a $g$-force can cause the cells to lyse and release the virus into the media.

8. Remove and save supernatant if further processing is required, and resuspend the cell pellet in $15 \mathrm{~mL} 10 \mathrm{mM}$ Tris- $\mathrm{HCl}, \mathrm{pH}$ 7.6. Store lysate at $-80^{\circ} \mathrm{C}$, until purification of the virus begins.

9. Freeze-thaw the pellet from $1010-\mathrm{cm}$ plates $3-4 \times$, alternating between an ethanol/dry ice bath and a $37^{\circ} \mathrm{C}$ water bath.

10. Spin at $1500 \mathrm{~g}$, for $15 \mathrm{~min}$ at $4^{\circ} \mathrm{C}$.

11. Save the lysate and wash the cellular debris pellet in $10 \mathrm{~mL} 10 \mathrm{~m} M$ Tris-Cl, $\mathrm{pH}$ 7.6. Spin as in previous step.

12. Save the supernatant and combine with the original $15 \mathrm{~mL}$ lysate from step $\mathbf{1 7}$, for a total amount of $25 \mathrm{~mL}$ lysate. Mix well, and save a $10-100-\mu \mathrm{L}$ aliquot for the in vitro assay for rAAV production.

13. Add $2000 \mathrm{U}$ DNaseI and $10 \mathrm{mg} / \mathrm{mL}$ RNase A (Ambion), to a final concentration of $0.2 \mathrm{mg} / \mathrm{mL}$ to the lysate and incubate in a $37^{\circ} \mathrm{C}$ water bath for $30 \mathrm{~min}$.

14. Add $25 \mathrm{~mL}$ (equal volume) of saturated $4 M$ ammonium sulfate to the lysate, incubate on ice for $20 \mathrm{~min}$ and spin at maximum speed for $15 \mathrm{~min}$.

15. Discard supernatant and resuspend pellet in $10 \mathrm{~mL}$ HEPES buffered saline (HBS), $\mathrm{pH}$ 7.8. Add and dissolve $6 \mathrm{~g} \mathrm{CsCl}$ to the resuspended pellet in $\mathrm{HBS}$ and place it into an SW-41Ti centrifuge tube or similar apparatus.

16. Spin gradient at $41,000 \mathrm{rpm}, 6^{\circ} \mathrm{C}$ for $24-48 \mathrm{~h}$. Harvest gradient in $1-\mathrm{mL}$ fractions, and perform an in vitro functional assay and dot blot on each fraction. rAAV is difficult to produce in titers that will constitute a visible band after ultracentrifugation; thus, the need for a diagnostic test for the presence of rAAV should be established.

17. The in vitro assay consists of $1-2 \mu \mathrm{L}$ of gradient fractions on 293 cells seeded in a 6-, 12-, or 24-well plate, in DMEM with $2 \%$ fetal bovine serum, in the presence of adenovirus, at a multiplicity of infection of 2 . The cells should be examined under a fluorescence microscope for GFP expression 24-48 h later.

18. Proceed to combine the positive rAAV fractions, based on the in vitro/dot blot results for a second SW-41Ti ultracentrifugation. Combine the fractions into one $12-\mathrm{mL}$ ultracentrifuge tube, and fill the tube with a stock of $1.37 \mathrm{~g} / \mathrm{mL} \mathrm{CsCl}$.

19. Ultracentrifuge the second gradient for $24-48 \mathrm{~h}$ at $41,000 \mathrm{rpm}, 6^{\circ} \mathrm{C}$. The virus can be more concentrated by allowing the spin to continue longer than $24 \mathrm{~h}$.

20. Harvest the gradient fractions in 1-mL vol, and assay again for the presence of rAAV.

21. Dialyze the positive fractions against HBS, $\mathrm{pH} 7.8$. Add $10 \%$ glycerol, then store at $-80^{\circ} \mathrm{C}$. Proceed to determine genomic titers by dot blot, using a kit such as the HybQuest system (Mirus), according to the manufacturer's recommendations. Comparison of the intensity of signals resulting from a known volume of viral stock to those of a known mass of psub201.EGFP.stop or WPRE plasmid used as a hybridization control allows the calculation of viral genomes per unit volume of stock. 


\subsection{Transduction of Cells with rAAV and Quantitation of GFP Expression}

1. Seed $10-\mathrm{cm}$ plates with 293 cells in DMEM supplemented with $10 \%$ fetal calf serum so that they will be $30 \%$ confluent at the time of transfection. Prior to transduction, replace the media with DMEM supplemented with $2 \%$ fetal calf serum. The reduced-serum media should be added at a volume just large enough to cover the cells; $4-6 \mathrm{~mL}$ should be sufficient in a $10-\mathrm{cm}$ plate.

2. Add equivalent MOI of the viral stocks with and without the WPRE to their respective plates, agitating gently. The authors found that the addition of 5000 viral genomes per target cell of stock was sufficient to render more than $90 \%$ of target 293 cells infected. After addition of viral stock, incubate the cells at $37^{\circ} \mathrm{C}$ $\left(5 \% \mathrm{CO}_{2}\right)$ for $4 \mathrm{~h}$ with occasional agitation, after which the media should be supplemented with additional fetal calf serum, to bring the serum content up to $10 \%$.

3. Depending on the goals of the experiment, the cells may be harvested and assayed for transient GFP expression at $48 \mathrm{~h}$ following transduction, or they may be selected in media containing $200 \mu \mathrm{g} / \mathrm{mL} \mathrm{G418} \mathrm{(Gibco)} \mathrm{for} \mathrm{3-4} \mathrm{wk} \mathrm{to} \mathrm{establish}$ stably transduced populations.

4. To prepare for quantification of GFP expression by flow cytometry, wash the cells once with PBS, and harvest in PBS $5 \mathrm{~m} M$ EDTA at a density of approx $2 \times$ $10^{6}$ cells $/ \mathrm{mL}$. To permit the exclusion of dead cells from the analysis, propidium iodide may be added to a final concentration of $5 \mathrm{~m} M$.

\section{Notes}

1. Expression plasmids should be selected using standard criteria for the generation of fusion proteins with GFP; the plasmid should contain a multiple cloning site 5' or 3' of GFP as appropriate to allow in-frame insertion of the gene to be studied within the message. An additional consideration when using the WPRE is that there must be a restriction enzyme site or sites suitable to allow the insertion of the $600 \mathrm{bp}$ WPRE sequence 3' of the gene of interest and the GFP open reading frames and their stop codon, but 5' of the polyadenylation sequence for the message.

2. These primers anneal to the first and last 24 bases of the WPRE sequence, encompassing bases 1093-1684. Desired restriction enzyme sequences should be appended to the $5^{\prime}$ end of each primer, to allow subsequent subcloning into expression vectors according to the criteria discussed in Methods.

3. The following general considerations should be taken into account when designing a cloning strategy that incorporates the WPRE into a plasmid expressing GFP fusion proteins:

a. The WPRE acts at the level of RNA, so it must be included in cis within the message it is to enhance. The WPRE must be in the sense orientation to function.

b. The WPRE is a roughly 600 base-long sequence; it should thus be inserted 3' of the GFP fusion protein gene to avoid reducing the efficiency of translation. Long 5' untranslated regions and structured RNA can reduce expression by perturbing ribosome function. 


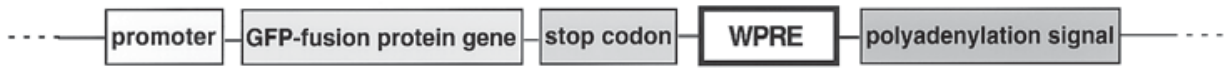

Fig. 3. Schematic illustrating correct placement of the WPRE in a construct expressing a GFP fusion protein. The WPRE should be included in the sense orientation within the message between the stop codon and polyadenylation signal of the gene of interest.

c. In its viral context the WPRE is located 139 bases from the noncanonical polyadenylation sequence. Constructs that place the WPRE 100-500 bases from the polyadenytion signal work well.

d. The WPRE should not be translated in order to avoid interference with the function of the fusion protein of interest. This requires that it be positioned 3 ' of the stop codon that terminates the translation of the fusion protein, but $5^{\prime}$ of the polyadenylation signal and related sequences that facilitate the 3 ' end formation of the message (Fig. 3).

4. The WPRE has been shown by this and other labs to enhance gene expression in the context of multiple genes, promoters, and polyadenylation sequences. The degree of enhancement will vary depending on the fusion protein expressed, the cells used, and other parameters of the expression system.

5. The full-length, functionally defined WPRE encompasses nearly $600 \mathrm{bp}$. If the size of the message is an issue, the WPRE sequence may be shortened by deleting bases 1504-1684, to yield a minimally diminished enhancement that varies by system.

6. If one intends to generate a fusion protein in which GFP is positioned N-terminal to the protein of interest, it will be useful to consider commercially available plasmids designed for this purpose. Such plasmids generally contain multiple cloning sites, following the GFP reading frame, permitting a choice of restriction enzyme sites sufficient to allow the insertion of the gene of interest and the WPRE, as well as an artificial stop codon between the gene to be studied and the WPRE. Such a stop codon can be synthesized with the appropriate overhanging ends, to take advantage of restriction sites available at this position.

\section{References}

1. Antoniou, M., Geraghty, F., Hurst, J., et al. (1998) Efficient 3'-end formation of human beta-globin mRNA in vivo requires sequences within the last intron but occurs independently of the splicing reaction. Nucl. Acids Res. 26, 721-729.

2. Nesic, D., Cheng, J., and Maquat, L. E. (1993) Sequences within the last intron function in RNA 3'-end formation in cultured cells. Mol. Cell Biol. 13, 3359-3369.

3. Ryu, W. S. and Mertz, J. E. (1989) Simian virus 40 late transcripts lacking excisable intervening sequences are defective in both stability in the nucleus and transport to the cytoplasm. J. Virol. 63, 4386-4394.

4. Donello, J. E., Beache, A. A., Smith, G. J., 3rd, et al. (1996) The hepatitis B virus posttranscriptional regulatory element is composed of two subelements. J. Virol. 70, 4345-4351. 
5. Donello, J. E., Loeb, J. E., and Hope, T. J. (1998) Woodchuck hepatitis virus contains a tripartite posttranscriptional regulatory element. J. Virol. 72, 5085-5092.

6. Fischer, U., Meyer, S., Teufel, M., et al. (1994) Evidence that HIV-1 Rev directly promotes the nuclear export of unspliced RNA. EMBO J. 13, 4105-4112.

7. Huang, Z. M. and Yen, T. S. (1994) Hepatitis B virus RNA element that facilitates accumulation of surface gene transcripts in the cytoplasm. J. Virol. 68, 3193-3199.

8. Malim, M. H., Bohnlein, S., Hauber, J., et al. (1989) The HIV-1 rev trans-activator acts through a structured target sequence to activate nuclear export of unspliced viral mRNA. Nature 338, 254-257.

9. Pasquinelli, A. E., Ernst, R. K., Lurd, E., et al. (1997) The constitutive transport element (CTE) of Mason-Pfizer monkey virus (MPMV) accesses a cellular mRNA export pathway. EMBO J. 16, 7500-7510.

10. Saavedra, C., Felber, B., and Izaurralde, E. (1997) The simian retrovirus-1 constitutive transport element, unlike the HIV-1 RRE, uses factors required for cellular mRNA export. Curr. Biol. 7, 619-628.

11. Huang, Z. M. and Yen, T. S. (1995) Role of the hepatitis B virus posttranscriptional regulatory element in export of intronless transcripts. Mol. Cell Biol. 15, 3864-3869.

12. Smith, G. J., III, Donello, J. E., Luck, R., et al. (1998) The hepatitis B virus posttranscriptional regulatory element contains two conserved RNA stem-loops which are required for function. Nucl. Acids Res. 26, 4818-4827.

13. Harris, M. E., et al. (2001) A cis-acting RNA element that increases expression by promoting longer polyadenylated tails, in preparation.

14. Huang, Y., Wimler, K. M., and Carmichael, G. G. (1999) Intronless mRNA transport elements may affect multiple steps of pre-mRNA processing. EMBO J. 18, $1642-1652$.

15. Loeb, J. E., Cordier, W. S., Harris, M. E., et al. (1999) Enhanced expression of transgenes from adeno-associated virus vectors with the woodchuck hepatitis virus posttranscriptional regulatory element: implications for gene therapy. Hum. Gene Ther. 10, 2295-2305.

16. Xiao, X., Li, J., and Samulski, R. J. (1998) Production of high-titer recombinant adeno-associated virus vectors in the absence of helper adenovirus. J. Virol. 72, 2224-2232.

17. Zufferey, R., Donello, J. E., Trono, D., et al. (1999) Woodchuck hepatitis virus post-transcriptional regulatory element (WPRE) enhances expression of transgenes delivered by retroviral vectors. J. Virol. 73, 2886-2892.

18. Samulski R. J., Chang L. S., and Shenk, T. (1987) A recombinant plasmid from which an infectious adeno-associated virus genome can be excised in vitro and its use to study viral replication. J. Virol. 61, 3096-3101. 